Pulsar Astronomy - 2000 and Beyond

ASP Conference Series, Vol. 202, 2000

M. Kramer, N. Wex, and R. Wielebinski, eds.

\title{
Interstellar scintillation of the radio source associated with the gamma ray burst of 8 May 1997
}

\author{
T.V. Smirnova and V.I. Shishov \\ Radio Astronomy Observatory of Astro Space Center, Lebedev Physical \\ Institute, Pushchino, Moscow region 142292, Russia
}

\begin{abstract}
We show that variability of the radio source associated with the gamma ray burst of May 8, 1997 can be explained by scintillations on interstellar plasma inhomogeneities in the weak regime at 4.86 and $8.46 \mathrm{GHz}$, and by the refractive component of saturated scintillations at $1.43 \mathrm{GHz}$. Cosmological distance of this source $R \cong 10^{28} \mathrm{~cm}$ is more preferable. The source has the size about $2 \mu$ arcsec at frequency 4.86 $\mathrm{GHz}$ and the velocity of spreading is about $25 \mu \mathrm{arcsec} /$ year.
\end{abstract}

The paper by Frail et al.(1997) reported the discovery of the variable radiosource VLA J065349.4+791619 within the error box of GRB970508 and coincident with the optical transient. The afterglow of this GRB revealed optical absorption lines at $\mathrm{z} \leq 0.835$ (Bloom et al. 1998), confirming the cosmological distance of the source. The goal of our paper is interpretation of observed variability of VLA J0653+79 on the base of modern data about interstellar medium and scintillations.

We analysed flux variations of radiosource VLA J065349.4+791619 at frequencies $8.46 \mathrm{GHz}$ and $4.86 \mathrm{GHz}$, based on the data of Frail et al.(1997) and Galama et al.(1998) at $1.43 \mathrm{GHz}$. Good enough correlation exists for these variations at both higher frequencies as with a short time ( $\leq 1$ day) as for more slower variations ( $\cong 30$ days). We devided the whole interval of observations on 2 parts. The first is up to June 9.74 ( $\sim 32$ days after burst) when the short time variability with a strong modulation was observed and second - up to August 4 when amplitude of variations was decreased. The modulation indexes calculated by us a.t both frequencies: $m_{4.86}=0.44$ and $m_{8.46}=0.32$ for the first interval and $m_{4.86}=0.22, m_{8.46}=0.2$ for the second one. We evaluated the time scale of variations at both frequencies as $t=1 / 3$ days in the first interval. Radioemission at $1.43 \mathrm{GHz}$ during the first 30 days was undetectable $(S<80 \mu J y)$. Calculated modulation index is $m_{1.43}=0.66$ after first 30 days of observations till the end. The time scale for the flux variations is about 10 days or less but more than 2 days. Flux variations at $1.43 \mathrm{GHz}$ doesn't correlate with variations at more higher frequencies. Radiosource VLA J0653+79 has a very close coordinats to PSR J0653+80, which has a dispersion mesure $D M=32.5 \mathrm{pc} / \mathrm{cm}^{3}$ and distance $\mathrm{R}=3 \mathrm{kpc}$. The value of critical frequency which separate two regims: weak and strong scintillations, $f_{c r}=3 \mathrm{GHz}$ at the distance $R_{0}=(2-3) \mathrm{kpc}$ in accordance to the dependence $f_{c r}(R)$ obtained by Malofeev et al.(1996). In the case of Kolmogorov spectrum $(n=11 / 3)$ for a weak scintillation we have: $m=\left(f_{c r} / f\right)^{\alpha}, \alpha=(n+2) / 4 \cong 1.4$. Characteristic time scale of scintillations is: $t_{0}=b_{f r} / V \propto 1 / \sqrt{(f)}$, where $b_{f r}$ is the Frenel zone size, $\mathrm{V}$ - the velocity of the 
line of sight. Taking $\mathrm{R}=3 \mathrm{kpc}$ we will have $t_{0}[\mathrm{~h}]=330(3 \mathrm{GHz} / \mathrm{f})^{0.5} / \mathrm{V}[\mathrm{km} / \mathrm{sec}]$. In the case of strong scintillation $\left(f<f_{c r}\right)$ there are two components: diffractive and refractive. The modulation index of diffractive one, $m_{d i f} \cong 1$. Using the experimental dependences of $m_{r e f}$, and the time scale, $T_{r e f}$, on DM at 610 $\mathrm{MHz}$ (Smirnova, Shisov \&Stinebring 1998) we have for $D M=30 \mathrm{pc} / \mathrm{cm}^{3}$ and Kolmogorov spectrum:

$$
\begin{aligned}
& \left.m_{r e f} \cong 0.4(f / 600) M H z\right)^{\gamma} \cong(f / 3 G H z)^{\gamma}, \gamma=(4-n)(n+2) /[2(n-2)]=0.56 \\
& T_{r e f}[\text { days }] \cong 30(f / 3 G H z)^{-\delta} / V[k m / s e c], \delta=n /(n-2)=2.2
\end{aligned}
$$

We have a good agreement between an experimental data and the theory predictions for dependences $m(f)$ and $t(f)$ if the source velocity is $30 \mathrm{~km} / \mathrm{sec}$ and flux variations are determined by weak scintillation regime at $4.86 \mathrm{GHz}$ and $8.46 \mathrm{GHz}$ and refractive scintillation at $1.43 \mathrm{GHz}$. To agree $\mathrm{m}(\mathrm{f})$ for the second interval we have to assume that the angular size of the source is increasing with increasing of the wavelength. In the first interval our source has an angular size which is about the Frenel zone size: $\varphi_{f r} \cong 2.1 \mu \operatorname{arcsec}$ at $4.86 \mathrm{GHz}$. The best agreement of observed flux variations with interstellar scintillation as a reason of them will be at $\varphi_{0}=5.8(3 G H z / f) \mu a r c s e c$.

Extragalactic nature of the source is more reliable. a) The distance to the source is $\mathrm{R}=10^{28} \mathrm{~cm}$. The flux of the source was changed for the time $\mathrm{T}=$ 30 days and it is the time of internal variability because it is not explained by interstellar scintillation. Assuming the relatevistic moving of material we have the upper value of the source angular size: $\varphi<\varphi_{\max }=c T / R \cong 2 \mu a r c s e c$. Of course the source with this size should scintillate. Increasing angular size in 2 times in a month is a consequence of relatevistic movements in the source. The linear size of this source sould be $l=\varphi R \cong 10^{17} \mathrm{~cm}$. If we assume that gamma emission originates in a compact region and then spread out on the whole volume of radiosource then the time for this should be less than 5 days and the visible velocity of shock spreading sould be about $7 \mathrm{c}$. b) The distance is $30 \mathrm{Mpc}$. In this case the size of the source is $l=10^{15} \mathrm{~cm}$. This size becomes 2 times more for the time 30 days, so velocity of disturbences should be $V=3800 \mathrm{~km} / \mathrm{sec}$. These parameters are in agree with SNR. If optical transient of this gamma ray burst is phisically connected with gamma and radio sources then variant a) is more preferable.

This work was supported by RFFI grant 97-02-17372 and INTAS-96-0154 project.

\section{References}

Bloom et al. 1998, ApJ, 507, L25

Frail, D.A. et al. 1997, Nature, 389, 261

Galama, T.J. et al. 1998, ApJ, 500, L101

Malofeev, V.M. et al. 1996, Astron.Astroph., 308, 180

Smirnova, T.V., Shishov, V.I. \& Stinebring, D.R. 1998, AZh, 42, 766 